\title{
Weekday and weekend patterns of physical activity and sedentary time among Liverpool and Madrid youth
}

\begin{abstract}
Introduction. Levels of physical inactivity and sedentary behaviour among English and Spanish youth are high and vary within different regions of each country. Little though is known about these during specific periods of the day. The purpose of this study was to describe PA and sedentary time during segments of the day and week and compare these critical contexts between youth in the Liverpool and Madrid areas of England and Spain, respectively.
\end{abstract}

Methods. PA was objectively assessed in 235 Liverpool and 241 Madrid youth (aged 10-14 years) who wore accelerometers for seven consecutive days. Minutes of sedentary time, moderate PA, vigorous PA, and moderate-to-vigorous PA were calculated for weekdays, weekend days, school time, non-school time, and after-school. Between country differences were analysed using ANCOVAs.

Results. Madrid youth spent significantly more time in sedentary activity than Liverpool counterparts. Madrid youth engaged in more minutes of MPA than Liverpool youth during weekdays, school time, and non-school time $(P<0.01)$. Liverpool children recorded more time in VPA than Madrid peers during week days and weekend days $(P<0.01)$, and during school time and after-school periods $(P<0.01)$. MVPA was significantly higher among Madrid youth during-non-school time $(P<0.01)$. Around $25 \%$ of all youth achieved recommended levels of moderate-to-vigorous PA.

Conclusion. Low levels of MVPA and systematic differences in sedentary time, MPA, and VPA exist between Liverpool and Madrid youth. Interventions targeted at the least active children during weekends, after-school, and non-school periods within the cultural contexts common to each city are required. 
Keywords. Accelerometer, moderate physical activity, vigorous physical activity, discretionary time, segmented day 


\section{Introduction}

The positive effects of physical activity (PA) on children and adolescents' health are well documented (Andersen, Riddoch, Kriemler, \& Hills, 2011; Hills, Andersen, \& Byrne, 2011) but globally most young people do not achieve the public health goal of at least 60 minutes per day of moderate-to-vigorous physical activity (MVPA) (Hallal, et al., 2012). Furthermore, physical inactivity becomes more prevalent from childhood through adolescence, and into adulthood, and is higher in girls than in boys (Hallal, et al., 2012). This worrying situation has led to physical inactivity being identified as the fourth major risk factor for non-communicable diseases (WHO, 2011). Low PA levels and increased time spent in sedentary pursuits are implicated in the growing prevalence of childhood overweight and obesity, rates of which are high in specific parts of Europe (Janssen, et al., 2005). Global trends in youth PA are also reflected in individual countries within Europe. Of the European countries ranked in the top 10 for youth overweight and obesity prevalence, England and Spain are among the largest in terms of populace and are contrasted by their geographical locations and climatic conditions (Janssen, et al., 2005). Analysis of objectively assessed PA among a nationally representative sample of English youth revealed that the proportion of boys and girls aged 4 to 10 years that achieved PA guidelines was $51 \%$ and $34 \%$, respectively (The NHS Information Centre, 2009). This fell to $7 \%$ of boys and $0 \%$ of girls aged 11 to 15 years (The NHS Information Centre, 2009). In both sexes sedentary time increased with age (The NHS Information Centre, 2009).

Nationally representative objectively assessed data of youth PA have not been gathered in Spain, but several regional studies have been conducted. For example, among 9-year old children from Madrid $60 \%$ of boys and $34.1 \%$ of girls achieved the PA guidelines, compared to $28.8 \%$ and $8.9 \%$ of 15 year olds, respectively, while sedentary time was highest among the older group (Aznar, et al., 2011). Furthermore, among 12-17 year olds from Zaragoza PA guidelines were achieved by $57.9 \%$ of boys and $23.6 \%$ of girls (Moliner-Urdiales, et al., 2009). 
Youth PA and sedentary time vary by periods within individual days (Gidlow, Cochrane, Davey, \& Smith, 2008) and between days of the week (Kristensen, et al., 2008). This is often due to the degree of organisational structure imposed, which can influence young people's activities. Structured and discretionary school day PA contexts such as physical education lessons (Fairclough \& Stratton, 2005) and recess periods (Stratton, Ridgers, Fairclough, \& Richardson, 2007) are important contributors to children's overall PA, while research has also highlighted the role of non-school time for PA participation (Fairclough, Beighle, Erwin, \& Ridgers, 2012). Moreover, young people's PA generally decreases at weekends compared to school days (Fairclough, Ridgers, \& Welk, 2012) but little is known about how weekend levels of PA differ between young people from different countries and cultures.

The school day structure and discretionary nature of non-school time can both enable and present barriers to PA (Fairclough, Beighle, et al., 2012). Thus, there is a need for research examining youth PA and sedentary time across different segments of the day and week. Comparing these types of data between countries can aid understanding of international variation in youth PA and sedentary behaviours. Liverpool and Madrid are large cities in England and Spain that are contrasted by their geographical locations, cultural practices, and climates. They are though also characterised by sub-optimal levels of youth PA and sedentary time, and excessive levels of child overweight and obesity. The purpose of this study was to describe PA and sedentary time during key segments of the day and week and compare these critical contexts between Liverpool and Madrid youth.

\section{Methods}

\section{Participants}

Schools were recruited from Liverpool, north-west England and from the Madrid Metropolitan area of Spain. Two primary schools and three high schools in Liverpool, and two primary schools and four high schools in Madrid were approached as convenience samples and 
agreed to participate in the study. In Liverpool 235 children (143 girls) returned written informed parental consent and assent to participate (Primary school Year 6; $n=73$ (aged 1011 years), High school Year 8; $n=82$ (aged 12-13 years), and Year 9; $n=80$ (aged 13-14 years)). In Madrid 241 children (130 girls) returned written informed parental consent and

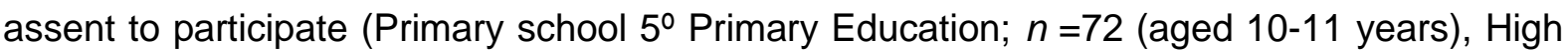
school $1^{\circ}$ Secondary Education; $n=88$ (aged $12-13$ years), and $2^{\circ}$ Secondary Education; $n$ $=81$ (aged 13-14 years)). Data were collected in one school per week between October and December 2009 (Liverpool) and between November 2009 and January 2010 (Madrid). Ethical approval was obtained from the Liverpool John Moores University Ethics Committee and San Carlos Clinic Hospital Clinical Research Ethics Committee, and all methods and procedures adhered to the Declaration of Helsinki.

\section{Instruments and procedures}

Anthropometry. Stature was measured to the nearest $0.1 \mathrm{~cm}$ using a portable stadiometer (Leicester Height Measure, Seca, Birmingham, UK/Barcelona, Spain). Body mass was measured to the nearest $0.1 \mathrm{~kg}$ using calibrated scales (Seca, Birmingham, UK/Barcelona, Spain). All measurements were taken by trained research staff with the children in light clothing and barefooted. Body mass index (BMI) was calculated (body mass $(\mathrm{kg}) /$ stature ${ }^{2}$ $\left.\left(\mathrm{m}^{2}\right)\right)$ and BMI z-scores were assigned to each child (Cole, Freeman, \& Preece, 1995). International Obesity Task Force age and sex-specific BMI cut-points were used to classify children as either normal-weight, overweight, or obese (Cole, Bellizzi, Flegal, \& Dietz, 2000).

Physical activity. PA was objectively measured every 5 seconds using ActiGraph accelerometers (GT1M, ActiGraph LLC, Pensacola, FL). Children were instructed to wear the ActiGraph over the right hip using a waist mounted nylon belt, from waking in the morning until bedtime for 7 consecutive days, including 2 weekend days. At the end of the data collection period ActiGraphs were downloaded and raw data files were checked for compliance to the monitoring protocol using Actlife 6 software (ActiGraph LLC, Pensacola, 
FL). Sustained 20 minute periods of zero counts suggested that the ActiGraph had been removed, and total 'missing' counts for those periods represented the duration that monitors were not worn (Catellier, et al., 2005). To reflect commonly observed differences in week day and weekend waking hours children were included in the data analysis if they wore the monitors for at least 540 minutes on week days and 480 minutes on weekend days, for a minimum of two week days and one weekend day. These inclusion criteria have previously shown acceptable reliability in similarly aged children (Mattocks, et al., 2008), and were achieved by 184 (78.3\%) and $192(79.7 \%)$ Liverpool and Madrid children, respectively. The 51 Liverpool and 49 Madrid children not meeting the minimum ActiGraph wear time criteria were excluded from subsequent data analyses.

ActiGraph cut points of 100 counts per minute, 2296 counts per minute, and 4012 counts per minute classified sedentary activity, moderate intensity physical activity (MPA), and vigorous intensity physical activity (VPA), respectively (Evenson, Catellier, Gill, Ondrak, \& McMurray, 2008). These cut-points were appropriate to the age group of interest and were recently highlighted as demonstrating acceptable classification accuracy across a range of intensities (Trost, Loprinzi, Moore, \& Pfeiffer, 2011). Mean minutes of sedentary time, MPA, and VPA were calculated by summing time spent in each activity threshold during each week day, weekend day, and during school time, non-school time, and after-school periods. In England children typically attend school from 09:00 to 15:30 with minimal variation to these timings. In Spain there is more variability with children typically attending primary and high school from 09:30 to $16: 00$ and from 08:30 to $14: 30$, respectively. Non-school time was defined as waking hours outside of the scheduled school day. After-school was defined as the three hour period immediately after the end of school (Atkin, Gorely, Biddle, Marshall, \& Cameron, 2008; Fairclough, Beighle, et al., 2012). During this period some school children walk, cycle, or are transported home or to another destination immediately that school ends, while others remain in school for a structured classroom activity or PA/sports club. As the timing and 
structure of the typical school days differed between countries, school day duration was recorded. The proportion of children who achieved PA recommendations of at least 60 minutes daily MVPA was calculated by identifying the children who achieved this recommendation on $\geq 50 \%$ of their valid measurement days (Olds, et al., 2007).

\section{Data analysis}

Primary school and high school data of boys and girls were combined to provide overall mean values for Liverpool and Madrid children and to optimise statistical power. Descriptive statistics for each city were calculated for all measured variables. The primary outcome variables were minutes of sedentary time, MPA, and VPA, and the independent variable was city. Separate ANCOVAs for times of the week (weekday, weekend day) and segments of weekdays (school time, non-school time, after-school) were computed for each dependent variable. PA variables that were excessively skewed underwent square root transformation. Subsequent Kolmogorov-Smirnov analyses showed that the distribution of all but four of these variables was not significantly different from a normal distribution ( $P>0.05)$. As ANOVA procedures are robust to deviations from normality (Tabachnick \& Fidell, 1996; Vincent, 1999) the planned ANCOVA analyses were conducted with all of the transformed data, which were back-transformed for presentation purposes. Analyses were adjusted for the effects of age, BMI z-score, gender, accelerometer wear time (where total days were analysed), and school day duration (for school day analysis). Chi-square tests compared the percentage of children achieving recommended levels of MVPA by city. Analyses were conducted using SPSS version 17 (SPSS Inc., Chicago, IL) with alpha set at $P<0.05$.

\section{Results}

Descriptive characteristics of the participants are presented in Table 1. Madrid youth had higher BMI values, BMI z-scores, and a greater proportion were classified as overweight/obese (36.4\% vs. $25.0 \%$ ). Weekday ActiGraph wear time duration was highest 
among Madrid youth, and school day duration was longest among Liverpool counterparts ( $P$ $<0.001$ ). PA levels did not differ between participants with $7,6,5,4$, or 3 days of valid data. There were no differences in stature, body mass, or BMI values between children who did and did not achieve ActiGraph inclusion criteria.

\section{Main analyses}

Main effects for sedentary time were evident in each time period $(P<0.01)$ with the exception of after-school $(P>0.05)$ (Figure 1$)$, indicating that Madrid youth spent significantly more minutes in sedentary activity than Liverpool counterparts. Madrid youth engaged in more minutes of MPA than Liverpool youth (Table 2) during weekdays, school time, and non-school time $(P<0.01)$. Liverpool children recorded more time in VPA than Madrid peers during week days and weekend days $(P<0.01)$, and during school time and after-school periods $(P<0.01)$. MVPA was significantly higher among Madrid youth during-non-school time $(P<0.01)$. Chi-square analyses revealed that $26.6 \%$ and $24.5 \%$ of Liverpool youth and $26.0 \%$ and $27.1 \%$ of Madrid youth achieved recommended levels of MVPA on week days and weekend days, respectively $(P>0.05)$. 


\section{Discussion}

Madrid children accumulated more sedentary time and MPA than Liverpool counterparts, but VPA was typically higher in the Liverpool sample. In both cities MVPA was low with most children not achieving recommended PA levels. Regardless of city, sedentary time and PA at all intensities was higher on weekdays than weekends.

A consistent finding was the between city differences in MPA and VPA. Madrid youth accumulated more MPA on weekdays, and during school and non-school time. Cultural and climatic differences may dictate specific factors which influence MPA. For example, Madrid youth may benefit from longer non-school hours, longer waking hours, and a dryer, warmer climate, compared to those in Liverpool. Combined, these factors may offer more conducive conditions for moderate intensity activities. The exception was the after-school period where MPA differences were negligible. In Liverpool, after-school PA and sport opportunities are frequent, particularly in high schools where specialist physical education teachers facilitate such activities. Moreover, a significant proportion of Liverpool children commonly walk home from school and in the process accumulate after-school MPA (Liverpool City Council, 2007). However, in Madrid high school youth in particular often eat lunch after school then start activities later rather than doing so immediately after the end of school. Furthermore, structured after-school PA is usually delivered away from school by private companies (Hardman, 2007) and so may not be as accessible as in Liverpool. Specific social, biological, economic, and environmental factors also likely influenced the PA outcomes but their measurement was beyond the scope of the study, and thus the findings should be interpreted with this in mind.

Liverpool youth engaged in most VPA possibly because opportunities for VPA are numerous through modifications to the physical environment (e.g., painted playgrounds (Ridgers, Stratton, Fairclough, \& Twisk, 2007)), extra-curricular PA sessions (TNS-BMRB, 2010), and 
the legacy of a UK government policy recommending at least two hours weekly curriculum physical education. High school physical education and extra-curricular PA are delivered by specialist physical education teachers who are more able than non-specialists to stimulate high activity levels (McKenzie, Sallis, Kolody, \& Faucette, 1997). Similarly, increasing numbers of primary schools employ specialist sports coaches to teach physical education and extra-curricular PA. Furthermore, at weekends a wide range of competition structures exist providing sporting opportunities that are not limited to traditional sports of football, rugby, and netball.. Competitive sports and team games in particular are known to engage children in higher levels of VPA than other forms of PA (Fairclough \& Stratton, 2005). During school time in Spanish cities like Madrid the adoption of sedentary behaviours is common, particularly among girls (Hernandez \& Valazquez, 2007). Madrid youth may be exposed to fewer structured PA initiatives than Liverpool counterparts which may reflect the differences in VPA. Moreover, in Spain extra-curricular and weekend PA opportunities are mainly focused on traditional, competitive sports that engage only a minority of youth (Hernandez, Ferrando, Quilez, Aragones, \& Terrenos, 2010). Non-school VPA was the same in both cities perhaps due to Liverpool youth compensating for higher VPA during school and afterschool by reducing activity later in the day. This 'activitystat' theory has been proposed in recent years (Wilkin, 2011) but there is insufficient evidence to fully support its existence (Reilly, 2011). It is more likely that environmental and cultural factors influenced non-school time VPA, such as the shorter daylight hours and less temperate weather conditions typical of Liverpool, and later evening meal times in Madrid which allow more time for activity in the latter part of the day.

This is the first study to contrast PA patterns of Liverpool and Madrid children, therefore direct comparisons with other studies are difficult to make. Sedentary time was 60-100 minutes/day lower than reported in the Health Survey for England (The NHS Information Centre, 2009), which used sedentary time cutpoints of 200 counts/minute rather than the broadly accepted 100 counts/minute that we used. Differences in sedentary time may 
therefore be minimal if the lower threshold were employed in both cases. Our Madrid sample's sedentary time was lower than the 500 minutes/day previously reported in adolescents from the city (Martinez-Gomez, Welk, Calle, Marcos, \& Veiga, 2009). As these youth were 3-5 years older than our sample the typically observed age-related increases in sedentary time (Aznar, et al., 2011; The NHS Information Centre, 2009) may provide some explanation of this difference. Youth sedentary time is observed to be a risk factor for various indicators of health (Tremblay, et al., 2011). In support of previous findings among Madrid youth, the high levels of sedentary time combined with higher BMI z-scores in our Madrid sample may place them at greater future cardiovascular health risk (Martinez-Gomez, et al., 2010).

Liverpool youth's weekday and weekend MVPA was low when compared to previous data from the city (Fairclough, Beighle, et al., 2012; Ridgers, Graves, Foweather, \& Stratton, 2011), and a nationally representative sample (The NHS Information Centre, 2009). The latter used an MPA count cutpoint of 2802 count/minute which would have produced more conservative MVPA levels than the threshold that we used (Evenson, et al., 2008). MVPA was substantially lower in our Madrid sample compared to other Madrid youth (Aznar, et al., 2011) and those from Zaragoza (Moliner-Urdiales, et al., 2009). It is unclear why the children in our study were less active but seasonal effects cannot be ruled out. Liverpool data were collected during autumn and winter when PA levels are generally lower due to cooler temperatures, increased rainfall, and shorter day light hours (Carson \& Spence, 2010). It is however, more difficult to assess whether seasonality affected the Madrid sample's PA, due to a lack of information about the timing of data collection in the available Madrid comparison studies (Aznar, et al., 2011; Moliner-Urdiales, et al., 2009). 
A consistent finding in both countries was lower PA at weekends. This has been attributed to shorter waking hours at weekends resulting in lower accelerometer wear time (The NHS Information Centre, 2009). Nevertheless, weekends provide more discretionary time which in theory can positively influence PA (Fairclough, Beighle, et al., 2012), but in reality some youth choose more sedentary pastimes (Fairclough, Boddy, Hackett, \& Stratton, 2009). Not only did overall MPA and VPA decrease at weekends but the contribution of MPA and VPA to total MVPA differed between countries. In the Liverpool sample MPA contributed $62 \%$ and $66 \%$, and VPA contributed $38 \%$ and $34 \%$ to weekday and weekend MVPA, respectively. Conversely, in the Madrid sample MPA contributed $72 \%$ and $77 \%$, and VPA contributed $28 \%$ and $23 \%$ to weekday and weekend MVPA, respectively. Thus, Madrid youth's VPA component was $10-11 \%$ smaller than that of the Liverpool group. This could have implications from a public health perspective because VPA is positively associated with cardiorespiratory fitness (Parikh \& Stratton, 2011), and inversely associated with adiposity (Parikh \& Stratton, 2011) and cardiometabolic risk (Gobbi, et al., 2012). We did not assess these health indicators but did observe between-city differences in BMI z-scores. Though causal mechanisms cannot be assumed, the findings tentatively suggest that the lesser contribution of VPA to the Madrid children's MVPA deny them health benefits associated with higher intensity PA. Support for this supposition is provided by Madrid research highlighting the superior role of VPA on obesity prevention compared to lower intensity PA (Moliner-Urdiales, et al., 2009), and associations between low levels of fitness, and high prevalence of cardiovascular risk (Ortega, et al., 2005).

This is the first study to compare Liverpool and Madrid children's objectively assessed PA patterns at different intensities and across different times of the week and periods of the day. The study adds to the literature on PA patterns among European children. Strengths were the use of an objective measure of PA, the application of appropriate PA intensity cutpoints, utilisation of a well established accelerometer data reduction protocol, and analyses which 
controlled for effects of some known confounders of children's PA. The study was limited by the cross sectional design which prohibited inferences about cause and effect. Other limitations included the use of convenience sampling from one specific city in each country which limited generalisations of the findings to all parts of England and Spain. Furthermore, use of single data collection phases also meant that the effects of seasonality were unaccounted for, and the sample size did not allow for age and gender differences to be explored without compromising statistical power. Accelerometers have inherent limitations related to wear during water-based activities, and accuracy during upper body and loadbearing PA, as well as PA performed on inclines, all of which combine to underestimate PA. However, this degree of measurement error is smaller than would be expected from selfreport questions. The absence of information about the contexts and settings where PA occurred (e.g., location, weather conditions, etc) was also a limitation. Activity diaries and observational methods would have provided temporal indications of the contexts of the children's PA and sedentary time. Thus, it is acknowledged that a range of uncontrolled variables may have influenced the results, but resource constraints meant that additional measures were beyond the scope of the study.

This study found low levels of MVPA and systematic differences in sedentary time, MPA, and VPA between Liverpool and Madrid children. VPA in particular was less prevalent across time points among Madrid youth. Considering the strong relationships between VPA, adiposity, fitness, and cardiometabolic risk this is a concern. Weekends, after-school, and non-school periods provide significant PA opportunities in both cities. There is a need to target interventions at the least active children during these periods of time within the cultural contexts of each city. 


\section{References}

Andersen, L. B., Riddoch, C., Kriemler, S., \& Hills, A. (2011). Physical activity and cardiovascular risk factors in children. British Journal of Sports Medicine, 45(11), 871-876.

Atkin, A. J., Gorely, T., Biddle, S. J. H., Marshall, S. J., \& Cameron, N. (2008). Critical hours: Physical activity and sedentary behavior of adolescents after school. Pediatric Exercise Science, 20, 446-456.

Aznar, S., Naylor, P. J., Silva, P., Pérez, M., Angulo, T., Laguna, M., et al. (2011). Patterns of physical activity in Spanish children: a descriptive pilot study. Child: Care, Health and Development, 37(3), 322-328.

Carson, V., \& Spence, J. C. (2010). Seasonal Variation in Physical Activity Among Children and Adolescents: A Review. Pediatric Exercise Science, 22, 81-92.

Catellier, D. J., Hannan, P. J., Murray, D. M., Addy, C. L., Conway, T. L., Yang, S., et al. (2005). Imputation of missing data when measuring physical activity by accelerometry. Medicine and Science in Sports and Exercise, 37, S555-S562.

Cole, T. J., Bellizzi, M. C., Flegal, K. M., \& Dietz, W. H. (2000). Establishing a standard definition for child overweight and obesity worldwide: international survey. British Medical Journal, 320, 1240-1244.

Cole, T. J., Freeman, J. V., \& Preece, M. A. (1995). Body mass index reference curves for the UK, 1990. Archives of Disease in Childhood, 73, 25-29.

Evenson, K. R., Catellier, D. J., Gill, K., Ondrak, K. S., \& McMurray, R. G. (2008). Calibration of two objective measures of physical activity for children. J Sports Sci, 26, 15571565.

Fairclough, S. J., Beighle, A., Erwin, H., \& Ridgers, N. D. (2012). School day segmented physical activity patterns of high and low active children. BMC Public Health, 12(1), 406. 
Fairclough, S. J., Boddy, L. M., Hackett, A. F., \& Stratton, G. (2009). Associations between children's socioeconomic status, weight status, and sex, with screen-based sedentary behaviours and sport participation. International Journal of Pediatric Obesity, 4, 299-305.

Fairclough, S. J., Ridgers, N. D., \& Welk, G. (2012). Correlates of children's moderate and vigorous physical activity during weekdays and weekends. Journal of Physical Activity and Health, 9, 129-137

Fairclough, S. J., \& Stratton, G. (2005). 'Physical education makes you fit and healthy'. Physical education's contribution to young people's physical activity levels. Health Education Research, 20, 14-23.

Gidlow, C. J., Cochrane, T., Davey, R., \& Smith, H. (2008). In-school and out-of-school physical activity in primary and secondary school children. Journal of Sports Sciences, 26, 1411-1419.

Gobbi, R. M., Davies, I. G., Fairclough, S. J., Mackintosh, K. A., Warburton, G. L., Stratton, G., et al. (2012). Clustered cardiometabolic risk, cardiorespiratory fitness and physical activity in 10-11 year old children. The CHANGE! Project baseline. Archives of Exercise in Health and Disease, 3, 207-213.

Hallal, P. C., Andersen, L. B., Bull, F. C., Guthold, R., Haskell, W., \& Ekelund, U. (2012). Global physical activity levels: surveillance progress, pitfalls, and prospects. Lancet, $380,247-257$.

Hardman, K. (2007). Current Situation and Prospects for Physical Education in the European Union. Brussels: European Parliament Directorate General Internal Policies of the Union.

Hernández, J. L., Velázquez, R. (2007). La Educación Física, los estilos de vida y los adolescentes: cómo son, cómo se ven, qué saben y qué opinan (Estudio de la población escolar y propuestas de actuación) [Physical Education, Lifestyles and Adolescents: How They Are, How They Look, What They Know and What They 
Think (Study of the School Population and Proposals for Action)]. Barcelona, España: Graó

Hernández, L. A., Ferrando, J. A., Quilez, J., Aragones, M., \& Terrenos, J. L. (2010). Análisis de la actividad física en escolares del medio urbano [Analysis of Physical Activity in Urban Schools]. Madrid: Consejo Superior de Deportes.

Hills, A. P., Andersen, L. B., \& Byrne, N. M. (2011). Physical activity and obesity in children. British Journal of Sports Medicine, 45, 866-870.

Janssen, I., Katzmarzyk, P. T., Boyce, W. F., Vereecken, C., Mulvihill, C., Roberts, C., et al. (2005). Comparison of overweight and obesity prevalence in school-aged youth from 34 countries and their relationships with physical activity and dietary patterns. Obesity Reviews, 6, 123-132.

Kristensen, P. L., Korsholm, L., Moller, N. C., Wedderkop, N., Andersen, L. B., \& Froberg, K. (2008). Sources of variation in habitual physical activity of children and adolescent: the European Youth Heart Study. Scandanavian Journal of Medicine and Science in Sports, 18, 298-308.

Liverpool City Council. (2007). SportsLinx Lifestyles Report. Liverpool: LCC.

Martinez-Gomez, D., Eisenmann, J. C., Gomez-Martinez, S., Veses, A., Marcos, A., \& Veiga, O. L. (2010). Sedentary Behavior, Adiposity, and Cardiovascular Risk Factors in Adolescents. The AFINOS Study. Revista Española de Cardiología, 63, 277-285.

Martinez-Gomez, D., Welk, G. J., Calle, M. E., Marcos, A., \& Veiga, O. L. (2009). Preliminary evidence of physical activity levels measured by accelerometer in Spanish adolescents: the AFINOS Study. Nutricion Hospitalaria, 24, 226-232.

Mattocks, C., Ness, A. R., Leary, S. D., Tilling, K., Blair, S. N., Sheild, J., et al. (2008). Use of accelerometers in a large field-based study of children: Protocols, design issues, and effects on precision. Journal of Physical Activity and Health, 5, S98-S111.

McKenzie, T. L., Sallis, J. F., Kolody, B., \& Faucette, F. (1997). Long term effects of a physical education curriculum and staff development program: SPARK. Research Quarterly for Exercise and Sport, 68, 280-291. 
Moliner-Urdiales, D., Ruiz, J. R., Ortega, F. B., Rey-Lopez, J. P., Vicente-Rodriguez, G., España-Romero, V., et al. (2009). Association of objectively assessed physical activity with total and central body fat in Spanish adolescents; The HELENA Study. International Journal of Obesity, 33, 1126-1135.

Olds, T., Ridley, K., Wake, M., Hesketh, K., Waters, E., Patton, G., et al. (2007). How should activity guidelines for young people be operationalised. International Journal of Behavioral Nutrition and Physical Activity, 4(43).

Ortega, F. B., Ruiz, J. R., Castillo, M. J., Moreno, L. A., Gonzalez-Gross, M., Warnberg, J., et al. (2005). Low Level of Physical Fitness in Spanish Adolescents. Relevance for Future Cardiovascular Health (AVENA Study). Revista Española de Cardiología, 58, 898-909.

Parikh, T., \& Stratton, G. (2011). Influence of intensity of physical activity on adiposity and cardiorespiratory fitness in 5-18 year olds. Sports Medicine, 41, 477-488.

Reilly, J. J. (2011). Can we modulate physical activity in children? International Journal of Obesity 36, 1266-1269.

Ridgers, N. D., Graves, L. E. F., Foweather, L., \& Stratton, G. (2011). Examining the influences on boys' and girls' physical activity patterns: The A-CLASS Project. Pediatric Exercise Science, 22, 638-650.

Ridgers, N. D., Stratton, G., Fairclough, S. J., \& Twisk, J. W. R. (2007). Children's physical activity levels during school recess: A quasi-experimental intervention study. International Journal of Behavioral Nutrition and Physical Activity, 4(19).

Stratton, G., Ridgers, N. D., Fairclough, S. J., \& Richardson, D. J. (2007). Physical activity levels of normal-weight and overweight girls and boys during primary school recess. Obesity, 15, 1513-1519.

Tabachnick, C. B., \& Fidell, L. S. (1996). Using Multivariate Statistics (3rd ed.). New York: Harper Collins.

The NHS Information Centre. (2009). Health Survey for England 2008. Physical activity and fitness (Vol. 1). London: The Health and Social Care Information Centre. 
TNS-BMRB. (2010). PE and Sport Survey 2009/10. London: Department for Education.

Tremblay, M., LeBlanc, A., Kho, M., Saunders, T., Larouche, R., Colley, R., et al. (2011). Systematic review of sedentary behaviour and health indicators in school-aged children and youth. International Journal of Behavioral Nutrition and Physical Activity, $8(1)$.

Trost, S. G., Loprinzi, P. D., Moore, R., \& Pfeiffer, K. A. (2011). Comparison of accelerometer cut-points for predicting activity intensity in youth. Medicine and Science in Sports and Exercise, 43, 1360-1368.

Vincent, W. (1999). Statistics in Kinesiology $\left(2^{\text {nd }}\right.$ ed). Champaign, IL: Human Kinetics.

WHO. (2011). Global status report on noncommunicable diseases 2010, from http://www.who.int/nmh/publications/ncd report full en.pdf

Wilkin, T. J. (2011). Can we modulate physical activity in children?No. International Journal of Obesity, 36, 1270-1276. 
Table 1. Descriptive characteristics of Liverpool and Madrid youth

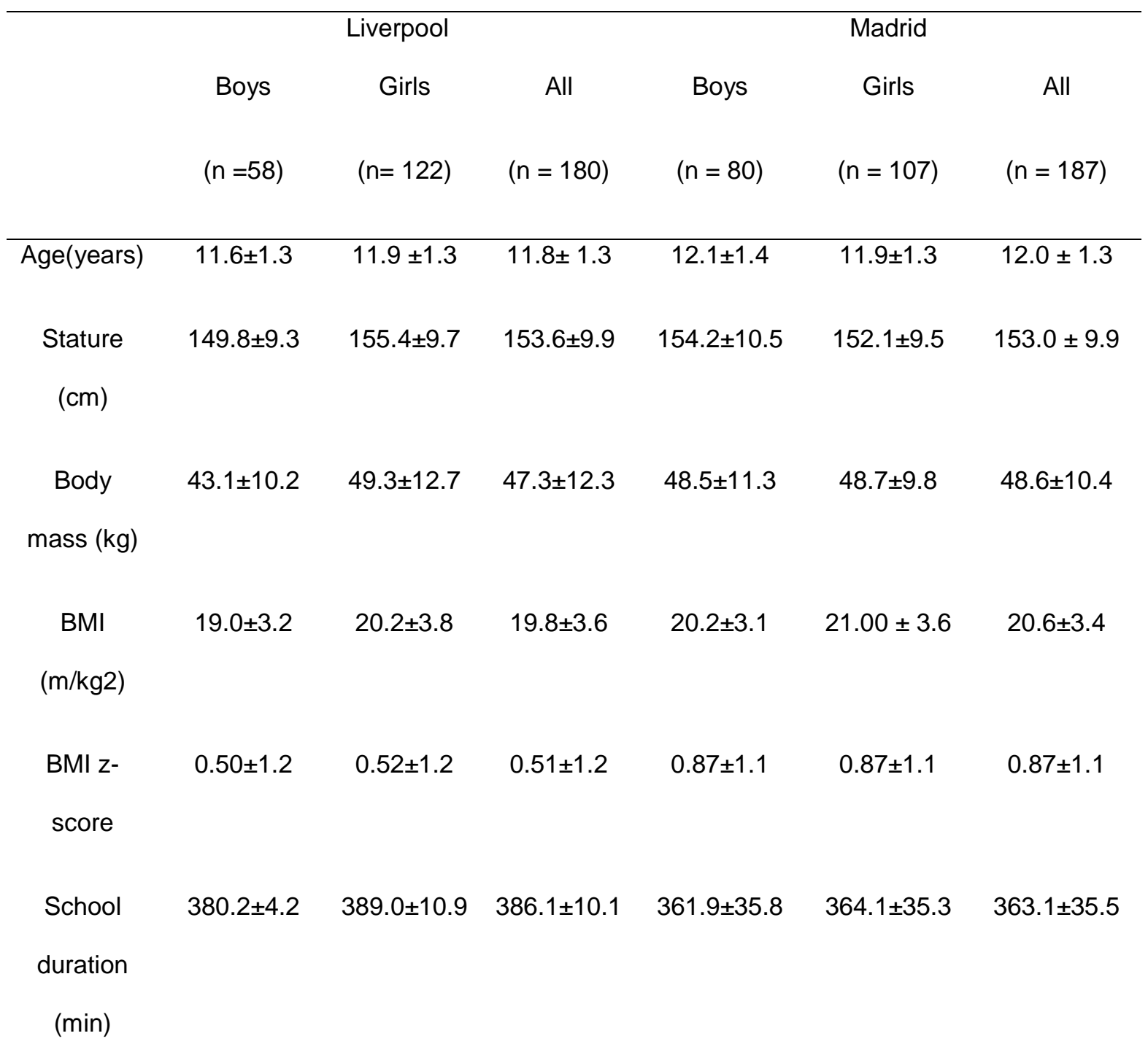

$\begin{array}{lllllll}\text { Week day } & 753.5 \pm 68.8 & 764.0 \pm 62.0 & 760.6 \pm 64.3 & 767.0 \pm 78.2 & 786.2 \pm 71.2 & 778.0 \pm 74.7\end{array}$ ActiGraph

wear time

(min)

Weekend $\quad 685.3 \pm 114.2 \quad 703.0 \pm 87.6 \quad 697.3 \pm 97.0 \quad 698.3 \pm 110.9 \quad 699.8 \pm 113.8 \quad 699.1 \pm 112.3$

ActiGraph

wear time

(min) 
Table 2. Adjusted mean ( \pm SE) minutes spent in MPA, VPA and MVPA

\begin{tabular}{|c|c|c|c|}
\hline & Liverpool & Madrid & \\
\hline & Mean $\pm S E$ & Mean $\pm S E$ & $P$ \\
\hline \multicolumn{4}{|l|}{ MPA (min) } \\
\hline Weekday & $30.1 \pm 0.9$ & $36.4 \pm 0.9$ & $<0.001$ \\
\hline Weekend day & $24.3 \pm 1.3$ & $26.4 \pm 1.3$ & 0.255 \\
\hline School time & $11.0 \pm 0.4$ & $14.3 \pm 0.4$ & $<0.001$ \\
\hline Non-school time & $17.3 \pm 0.7$ & $23.8 \pm 0.7$ & $<0.001$ \\
\hline After-school & $9.3 \pm 0.4$ & $10.0 \pm 0.4$ & 0.212 \\
\hline \multicolumn{4}{|l|}{ VPA (min) } \\
\hline Weekday & $18.4 \pm 0.9$ & $14.3 \pm 0.9$ & 0.001 \\
\hline Weekend day & $12.7 \pm 0.7$ & $8.1 \pm 0.7$ & $<0.001$ \\
\hline School time & $8.6 \pm 0.4$ & $4.7 \pm 0.3$ & $<0.001$ \\
\hline Non-school time & $9.7 \pm 0.7$ & $9.7 \pm 0.7$ & 0.980 \\
\hline After-school & $5.4 \pm 0.4$ & $3.8 \pm 0.4$ & 0.003 \\
\hline \multicolumn{4}{|l|}{ MVPA (min) } \\
\hline Weekday & $48.6 \pm 1.5$ & $50.6 \pm 1.5$ & 0.355 \\
\hline Weekend day & $36.5 \pm 1.7$ & $32.3 \pm 1.7$ & 0.088 \\
\hline School time & $19.6 \pm 0.7$ & $19.0 \pm 0.6$ & 0.504 \\
\hline
\end{tabular}




\begin{tabular}{llrl} 
Non-school time & $27.1 \pm 1.2$ & $33.5 \pm 1.2$ & $<0.001$ \\
After-school & $14.5 \pm 0.6$ & $13.8 \pm 0.6$ & 0.390 \\
\hline
\end{tabular}

Note MPA = moderate physical activity; VPA = vigorous physical activity; MVPA = moderate-tovigorous physical activity 
Figure legend

Figure 1. Mean ( \pm SE) sedentary time for time of the week (weekday, weekend day) and segments of weekdays (school time non-school time, after school). 